\title{
A CONTEXT-AwARE STRATEgY FOR MOBILE SERVICES IN M-COMMERCE
}

\author{
Kuen-Liang Sue \\ Department of Information Management, National Central University Taoyuan, Taiwan
}

\begin{abstract}
Due to mobile communication technologies, the novel mobile commerce is coming true. The context aware service is one of the most potential mobile commerce. The data about mobile users and their surroundings are recognized and collected, which are used to provide the services they may be interested. Fuzzy logical processing model are utilized to make basic data correspond to the mobile users' high-level information, so the recognition of their behaviours and situations could be inferred in our research. The numerical examples are also illustrated in our scheme. Based on the results of our activity recognition mechanism, it is possible to automatically discover and transmit the contents or services that users require in specific scenarios. Therefore, more and more novel mobile applications are coming true. To sum up, the proposed scheme is supposed to enhance context-aware services and provide more attractive life styles.
\end{abstract}

\section{KEYWORDS}

Mobile commerce, Context-aware service, Activity recognition

\section{INTRODUCTION}

Fourth Generation (4G) and other mobile technologies are well-developed and smart phones become more and more available to all. Therefore, people could have a modern living via the development of mobile communication networks. The mobile services based on the cellular networks have become a novel tendency, so mobile devices with services are becoming essential for smart life styles. Furthermore, more and more attractive mobile services are developed and make a new commercial style, which are call mobile commerce (i.e. M commerce).

Nowadays, mobile users could widely receive mobile applications which is becoming a popular business and creating the opportunities for mobile commerce due to the widespread of mobile computing. The context-aware service is one of the important mobile applications. Context aware applications are provided to the user by discovering and utilizing contextual information that characterizes the user's situation. Hence, if the context-aware application gets well developed, users' real-time demands or wish could be satisfied immediately and automatically. For example, when people are shopping in supermarket or department store, they can automatically get the digital advertisement or promotion information.

The context aware attributes and its framework should be well-organized to store, manage and utilize the context-aware information. Therefore, this research aims to estimate the mobile user's scenario by using basic sensor's data. To closely meet the requirement in real world, the proposed scheme could be adaptable by setting proper input or output items and has good flexibility. Most importantly, the concept of context-aware services could be realized through the novel strategy in the paper. 


\section{RELATED WORKS}

The location-based service is a well-known mobile service, which is one type of context-aware services. In brief, the context-aware service means a smart service providing according to the information about the mobile user's surroundings collected widely and automatically. The suggested services are judged to be attractive to the user under such scenario [1]. Hence, how to design the framework to support the smart promotion would be a key job. Moreover, to realize smart computing anytime and anywhere, a novel information technology called ubiquitous computing become hot issue in network fields[2]. This is an important field to support the oncoming context-aware service and mobile commerce [3].

Korpipaa et al. designed the context-aware attributes with the organized structure to manage the context-aware information systematically [4]. In addition, they classified the factors in an environment into sound, light, temperature and humidity and measured these value. Thus it is possible to obtain the high-level information from those basic factors. Whenever a user wants to query high-level contents or service providers would like to push services to the user, the context manager would reply to them according to the reasoning situation based on the sensing values of sensors. The progress for implementing such smart scenario is still an important issue on going.

Moreover, Mühlenbrock et al. tried to develop applications for the support of workplace interactions [5]. They utilized laptop, handset, personal digital assistant, ambient sound, and user feedback to collect basic sensor data. The activity, availability and inputs are also defined in their contribution. Furthermore, Azkune and Almeida proposed an unsupervised recognition technique [6]. Their study enabled users to label anonymous activity events by showing contextual information gained from the sensors in house.

Due to the literature survey concluded above, a smart approach to estimate high-level contents via the sensing value of sensors will definitely become the key point of developing the context aware service. To achieve goal, the raw data measured by surrounding sensor must be used to infer the user's need a concluding and reasoning mechanism. It needs to combine various factors related with the surroundings. In other words, an inferential strategy is required to estimate a user's uncertain situation. The estimation could be solved by means of fuzzy logic. Therefore, it is feasible to take advantage of fuzzy logic for reasoning context-awareness. The well-designed model with fuzzy sets will be able to match the requirement to estimate human behaviour in realworld [7].

Based on spatial reasoning decision support, the fuzzy logic has also been used for location tracking [8] [9]. Wu et al. analysed the multiple attributes of the product with respect to the consumer's desire by a fuzzy approach and classified these products into different levels of preference [10]. The proposed strategy in our investigation uses fuzzy logic to compute and get flexible high-level contents from raw data. The activity recognition means to guess or make sure what activity people are engaging in. Based on fuzzy logic approach a flexible strategy for achieving the activity recognition is presented in this paper.

\section{The Framework For CONTEXT-WARE SERVICE}

This study is constructed based on a context-aware framework. A mobile station (MS) which is usually a handset or a pad with a mobile user must be served under a base station in some cell and could roam to different cells. A base station controller (BSC) will coordinate different BSs to support mobility of MS. Moreover, a mobile switching centre (MSC) manages different BSC and mobile databases to provide roaming in a mobile network [11]. To provide context-aware 
services, a context server is collaborated with the BSC. To enables a MS know its own location, the location information about the user can estimated from MS's located cell or collected from Global Positioning System (GPS) as necessary.

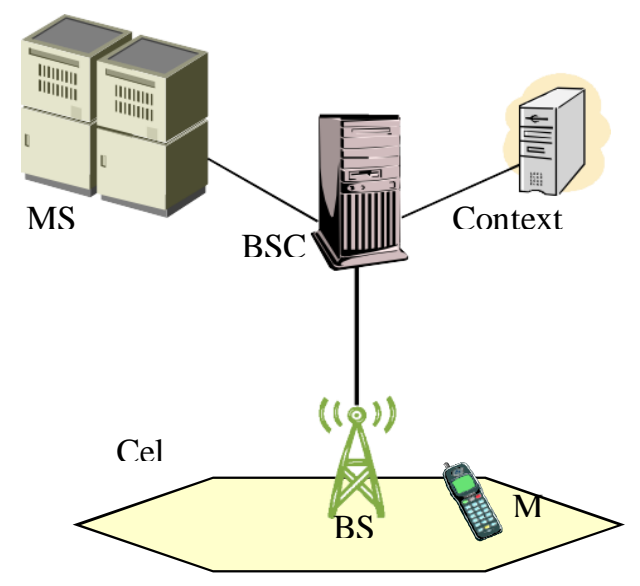

Figure 1. Framework of context-aware service.

Various data of context types are assembled by the context server. Moreover, the values of these data are converted to the corresponding membership degrees. Accordingly, activity recognition is preceded in the next step and the context server is engaged to provide suitable services in the estimated scenario. The proposed context-aware framework in the study is shown as Figure 1. It is necessary to define the input attributes for users' activity recognition in a mobile environment, including background noise, level of positioning signal, moving speed, working circumstance, and usage frequency of device. The scenario of a user will be reasoned based on these attributes. Furthermore, if a user feedback mechanism is also considered, it is possible to adjust and rebuild the membership degrees as well as the concrete rule base. By the way, a more and more accurate model through continuous training and adjustment can be constructed on the context server.

To sum up, a novel mechanism of recognizing context-aware information from the basic sensors data is proposed in this study. From the viewpoint of the mobile service provider, it becomes feasible to recognize a user's scenario and provide appropriate context-aware applications through the proposed mechanism.

\section{The Proposed SCHEME}

The processes in the proposed scheme contain basic context types, membership functions and fuzzy sets. It also involves the quantitative method of background values in the real world and complex messages for determining the information contents to apply to mobile services finally. For the conciseness of description, we will use the notation in Table 1.

\subsection{Basic Context Types}

The context-aware system would like and is able to know many basic context types via sensors laid out in mobile communication systems and personal mobile devices. The proposed mechanism utilizes the values of basic context types as input, which includes background noise, strength level of positioning signal, user's moving speed, working circumstance, and usage frequency of device. A set $\mathrm{U}$ is used to indicate these basic context types as follows:

$$
\mathrm{U}=\left\{\mathrm{u}_{1}, \mathrm{u}_{2}, \mathrm{u}_{3}, \mathrm{u}_{4}, \mathrm{u}_{5}\right\}
$$


Table 1. Definition of notations

\begin{tabular}{|l|l|}
\hline Notation & Description \\
\hline $\mathrm{u}_{1}$ & Background noise \\
\hline $\mathrm{u}_{2}$ & Level of positioning signal \\
\hline $\mathrm{u}_{3}$ & User's moving speed \\
\hline $\mathrm{u}_{4}$ & Working circumstance \\
\hline $\mathrm{u}_{5}$ & Usage frequency of device \\
\hline $\mathrm{v}_{1}$ & Low membership degree \\
\hline $\mathrm{v}_{2}$ & Moderate membership degree \\
\hline $\mathrm{v}_{3}$ & High membership degree \\
\hline $\mathrm{W}_{1}$ & Weight sets for location \\
\hline $\mathrm{W}_{2}$ & Weight sets for movement \\
\hline $\mathrm{W}_{3}$ & Weight sets for user task \\
\hline $\mathrm{W}_{4}$ & Weight sets for user situation \\
\hline
\end{tabular}

\subsection{Membership Functions}

In addition to the input values, each basic context type has its own corresponding membership function. Except the pure number of membership degree, it is important to pay more concern about the relation between all membership degrees.

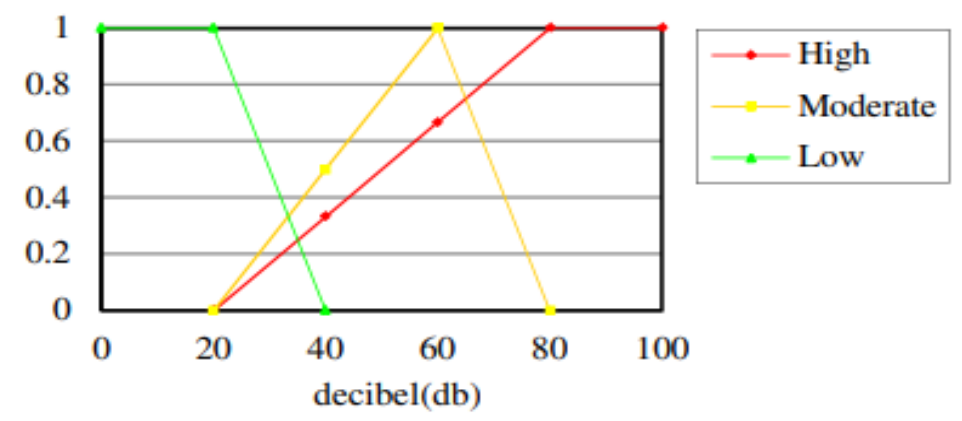

Figure 2. Membership function $\mu_{1}(\mathrm{x})$ for background noise

Standardization is an important step with membership function, which can avoid the effect of the extreme value no matter too large or small. For example, the membership function of background sound $\mu_{1}(\mathrm{x})$ is shown in Figure 2. The high, moderate and low membership degrees are defined as $\mathrm{v}_{1}, \mathrm{v}_{2}$ and $\mathrm{v}_{3}$ respectively in light of every membership function. The fuzzy set $\mathrm{V}$ formed by them is shown as follows:

$$
\mathrm{V}=\left\{\mathrm{v}_{1}, \mathrm{v}_{2}, \mathrm{v}_{3}\right\}
$$

\subsection{High-Level Context Types and Weights}

The high-level context types are derived from basic context data, which are the processing result and outputs from the proposed mechanism. For high-level context type, a user's situation could be classified into four categories of characteristics. For instance, the second one is moving speed. 
The possible states include still, walk, running, bicycle and fast vehicle. Each category of output has its own weight set. As shown in a weight set $\mathrm{W}_{\mathrm{i}}, \mathrm{x}_{1}$ represents the related level indicating the relationship for $\mathrm{u}_{1}$. The second one is $\mathrm{x}_{2}$ representing the corresponding level for $\mathrm{u}_{2}$ (i.e., the relationship for $\mathrm{u}_{2}$ ). The rest can be deduced by analogy, i.e. $\mathrm{x}_{\mathrm{k}}$ for $\mathrm{u}_{\mathrm{k}}, \mathrm{k}=1,2,3,4$, 5. In other words, weights are determined based on the levels of relationship between set $U$ and high-level context types. Hence, $\mathrm{W}_{\mathrm{i}}$ is defined as formula (3).

$$
\mathrm{W}_{\mathrm{i}}=\left\{\mathrm{x}_{1}, \mathrm{x}_{2}, \mathrm{x}_{3}, \mathrm{x}_{4}, \mathrm{x}_{5}\right\} \quad \text { where } \mathrm{i}=1,2,3,4 \text { and } \mathrm{x}=\mathrm{a}, \mathrm{b}, \mathrm{c}, \mathrm{d}
$$

Besides, the corresponding formulas for each high-level context type are shown as follows:

$$
\begin{aligned}
& \mathrm{W}_{1}=\left\{\mathrm{a}_{1}, \mathrm{a}_{2}, \mathrm{a}_{3}, \mathrm{a}_{4}, \mathrm{a}_{5}\right\} \\
& \mathrm{W}_{2}=\left\{\mathrm{b}_{1}, \mathrm{~b}_{2}, \mathrm{~b}_{3}, \mathrm{~b}_{4}, \mathrm{~b}_{5}\right\} \\
& \mathrm{W}_{3}=\left\{\mathrm{c}_{1}, \mathrm{c}_{2}, \mathrm{c}_{3}, \mathrm{c}_{4}, \mathrm{c}_{5}\right\} \\
& \mathrm{W}_{4}=\left\{\mathrm{d}_{1}, \mathrm{~d}_{2}, \mathrm{~d}_{3}, \mathrm{~d}_{4}, \mathrm{~d}_{5}\right\}
\end{aligned}
$$

\subsection{Fuzzy Logical Operation}

To start with the set $U$ and set $\mathrm{V}$ can be now gained from the definitions of formula (1) and (2). Secondly, the matrix $\mathrm{R}$ is listed for the following operation step:

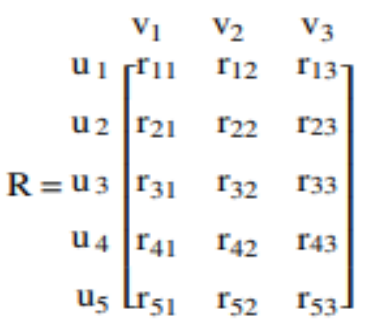

The matrix element $\mathrm{r}_{\mathrm{kj}}$ represents a value which some basic context element $\mathrm{u}_{\mathrm{k}}$ (e.g., $\mathrm{u}_{1}$ ) corresponds with some moderate membership degree $v_{j}\left(\right.$ e.g., $\left.v_{2}\right)$ in the membership function. A composition operation on fuzzy relations is performed, which aims to associate the related sets. It operates with a given fact to produce an output which makes the decision in a fuzzy way. The operative formula is as follows:

$$
\mathrm{o}_{\mathrm{ij}}=\bigvee_{\mathrm{k}=1}^{\mathrm{n}}\left(\mathrm{x}_{\mathrm{ik}} \wedge \mathrm{r}_{\mathrm{kj}}\right)
$$

\subsection{Outputs}

The three formulas (3), (4) and (6) can be used to compute and know some characteristic like user situation whenever we need to. Let the set Wi make a composition with matrix R accordingly, and then an output set is generated:

$$
\begin{aligned}
& \mathrm{O}_{\mathrm{i}}=\mathrm{W}_{\mathrm{i}} \times \mathrm{R} \\
& =\left[\begin{array}{lllll}
\mathrm{x}_{1} & \mathrm{x}_{2} & \mathrm{x}_{3} & \mathrm{x}_{4} & \mathrm{x}_{5}
\end{array}\right]\left[\begin{array}{ccc}
\mathrm{r}_{11} & \mathrm{r}_{12} & \mathrm{r}_{13} \\
\mathrm{r}_{21} & \mathrm{r}_{22} & \mathrm{r}_{23} \\
\mathrm{r}_{31} & \mathrm{r}_{32} & \mathrm{r}_{33} \\
\mathrm{r}_{41} & \mathrm{r}_{42} & \mathrm{r}_{43} \\
r_{51} & \mathrm{r}_{52} & \mathrm{r}_{53}
\end{array}\right]
\end{aligned}
$$




$$
=\left[\begin{array}{lll}
011 & 012 & 013
\end{array}\right]
$$

Hence, we can easily get the vectors $\mathrm{O}_{\mathrm{i}}$ through the computation. As if some mobile nodes have the similar $\mathrm{O}_{\mathrm{ij}}$, the users of these devices will be considered engaging in the similar/same activity or situation. More details will be shown on the strength of those examples in Section 5.

Most important, the classification can be obtained through the mechanism. The rule base shown in Table 2 is necessary to conclude the classification. Therefore, the classification result about various characteristics can be generated. The parameter $\mathrm{o}_{\mathrm{ij}}$ will be in connection with the composition of inputs. Because they conform to certain tendencies, the classification can be realized. In addition, a prototype of rule base is built up and the adjustment of classification could be emended via the user feedback when a system is implemented.

The value of parameter $\mathrm{O}_{\mathrm{ij}}$ is connected with the composition of inputs. For instance, suppose $\mathrm{W}_{2}$ $=\{0.2,0.5,0.8,0,0\}$ is fitting to a crowd scenario. As the parameters of $\mathrm{O}_{2}$ fits in with the following relationship: $\mathrm{O}_{13}>\mathrm{O}_{12} \wedge \mathrm{O}_{12}>\mathrm{O}_{11}$, it indicates that the tendency level for background noise, user's moving speed, and usage frequency of device is strong. Thus the user might be moving at high speed by taking a vehicle. If all weight set $\mathrm{W}_{\mathrm{i}}$ and $\mathrm{R}$ are known, the full result $\mathrm{O}$ $=\left\{\mathrm{O}_{1}, \mathrm{O}_{2}, \mathrm{O}_{3}, \mathrm{O}_{4}\right\}$ can be produced by simple computation.

Table 2. The Rule Base

\begin{tabular}{|l|l|l|l|l|l|}
\hline Types & Logical expression & Location $\left(\mathrm{O}_{1}\right)$ & Movement $\left(\mathrm{O}_{2}\right)$ & User task $\left(\mathrm{O}_{3}\right)$ & User situation $\left(\mathrm{O}_{4}\right)$ \\
\hline I & $011 \geq 012 \wedge 012 \geq 013$ & deep indoor & still & deep rest & very fine \\
\hline II & $011 \geq 013 \wedge 013>012$ & indoor & walk & rest & fine \\
\hline III & $012>011 \wedge 011>013$ & half-indoor & fast walk & available & ordinary \\
\hline IV & $012>013 \wedge 013>011$ & half-outdoor & run & active & complex \\
\hline V & $013>011 \wedge 011>012$ & open & vehicle & busy & disorder \\
\hline VI & $013>012 \wedge 012>011$ & very open & fast vehicle & extremely busy & extremely disorder \\
\hline
\end{tabular}

\subsection{Training}

By taking advantaging of training data, excellent weights as relying on the weight decision approach can be generated. In order to estimates the similarity, the similar degree is innovated, whose definition is shown as formula 7 . Both A and B represent fuzzy sets. The notations $\bullet$ and $\oplus$ means inner product, and outer product respectively.

$$
(\mathrm{A}, \mathrm{B})=\frac{1}{2}(\mathrm{~A} \cdot \mathrm{B})+\overline{(\mathrm{A} \oplus \mathrm{B})}
$$

Suppose that some output $\mathrm{O}_{\mathrm{i}}=\left[\mathrm{o}_{11}, \mathrm{o}_{12}, \mathrm{o}_{13}\right]$ and matrix $\mathrm{R}$ are known, it is possible to draft $\mathrm{n}$ kinds of probable weight sets $\left(\mathrm{W}_{\mathrm{i}}\right)_{\mathrm{t}}, \mathrm{t}=1 \sim \mathrm{n}$. Each of them does fuzzy composition with $\mathrm{R}$, respectively. Then, we acquire $\left(\mathrm{O}_{\mathrm{i}}\right)_{\mathrm{t}}$ and compute the approaching degree between $\left(\mathrm{O}_{\mathrm{i}}\right)_{\mathrm{t}}$ and $\mathrm{O}_{\mathrm{i}}$. The approaching degree 1 means most similar. Oppositely, degree 0 represents most dissimilar. A weight set with highest approaching degree which is the best weight set candidate should be selected.

\section{Numerical AnAlysis}

The numerical examples to illustrate how the proposed scheme works are shown in the section. Thirty students are observed for their work schedule and usage frequency of device in the 
International Journal of Database Management Systems (IJDMS) Vol.12, No.3, June 2020

example. The strength level of positioning signal and background noise are referred to the real values measured in the campus and its neighbourhood. The range of user's moving speed for walking and vehicle are measured respectively.

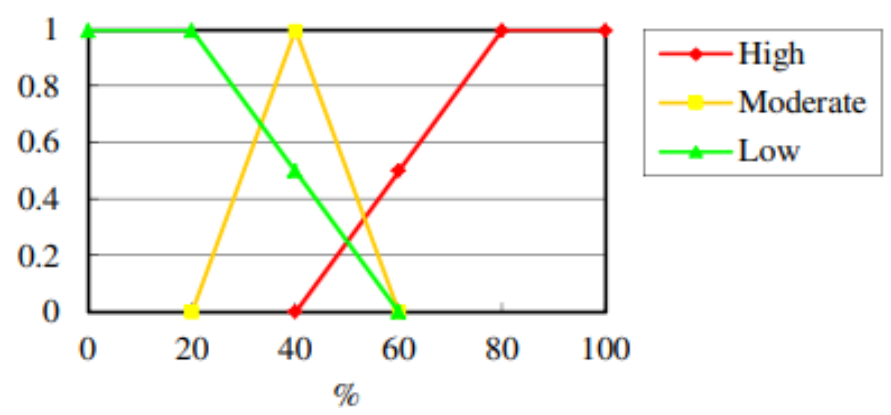

Figure 3: Membership function $\mu_{2}(\mathrm{x})$ for level of positioning signal

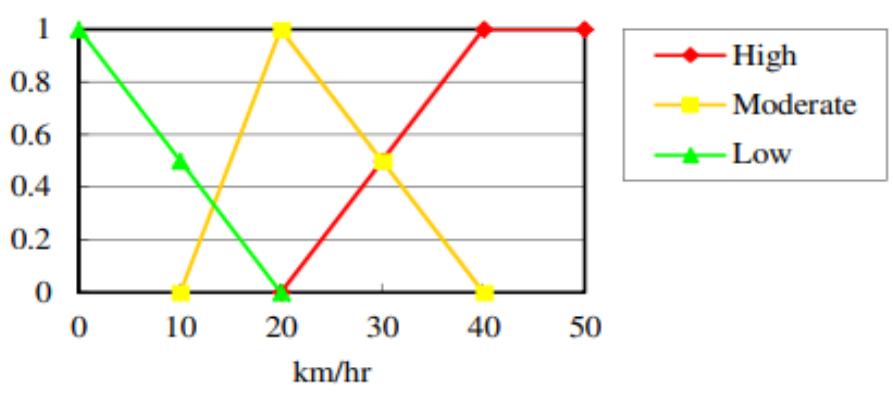

Figure 4: Membership function $\mu_{3}(\mathrm{x})$ for user's moving speed

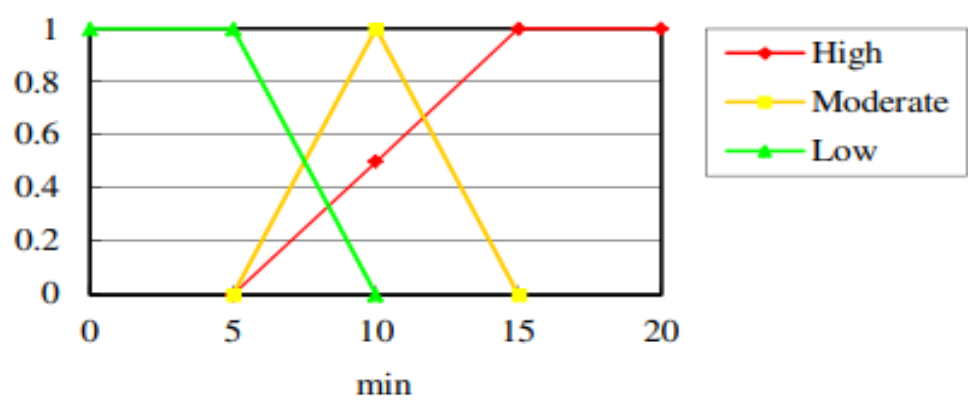

Figure 5: Membership function $\mu_{4}(\mathrm{x})$ for working circumstance

The membership functions from $\mu_{1}(\mathrm{x})$ to $\mu_{5}(\mathrm{x})$ are designed accordingly and shown in Figure 2 to Figure 6. It should be noted that the usage frequency of device expresses the minutes in the latest one hour. Harikesh et al. demonstrated how the membership function is generated from the realistic numerical data [12].

\subsection{Examples for classification}

A numerical example is illustrated in this subsection. For the characteristic classification, we set up weight sets as follows:

- Background weight sets $\mathrm{W}_{1}=\{0.1,0.8,0.1,0,0\}$

- Movement weight sets $\mathrm{W}_{2}=\{0.2,0,0.8,0,0.5\}$

- User task weight sets $\mathrm{W}_{3}=\{0.2,0,0.1,0.8,0.3\}$ 
- User situation weight sets $\mathrm{W}_{4}=\{0.7,0,0.3,0.1,0.2\}$

Suppose that we want to know the background around a user $\mathrm{j}$. To start with, the proposed scheme computes the composition of $\mathrm{W}_{1}$ and $\mathrm{R}_{\mathrm{j}}$, i.e. $\mathrm{O}_{1}$. The following steps can be deduced by analogy. As a result, the resulting output is $\mathrm{O}_{\mathrm{Rj}}=\left\{\mathrm{O}_{1}, \mathrm{O}_{2}, \mathrm{O}_{3}, \mathrm{O}_{4}\right\}$. For example, the complete data of five mobile nodes are given in Table 3.

Table 3: Numerical examples of 5 mobile nodes

\begin{tabular}{|c|c|c|c|c|c|}
\hline & $\mathrm{u}_{1}(\mathrm{db})$ & $\mathrm{u}_{2}(\%)$ & $\mathrm{u}_{3}(\mathrm{~km} / \mathrm{hr})$ & $\mathrm{u}_{4}$ (o'clock) & $\mathrm{u}_{5}(\min )$ \\
\hline MS1 & 80 & 40 & 16 & 16 & 15 \\
\hline MS2 & 40 & 40 & 10 & 12 & 10 \\
\hline MS3 & 80 & 70 & 36 & 20 & 5 \\
\hline MS4 & 20 & 20 & 0 & 22 & 0 \\
\hline MS5 & 50 & 60 & 5 & 8 & 0 \\
\hline
\end{tabular}

$\mathrm{O}_{1}$ of the mobile node MS1 is illustrated as an example. The variable $\mathrm{u}_{1}$ is 80 decibel, so membership degree $\mathrm{v}_{1}$ is $0, \mathrm{v}_{2}$ is 0 , and $\mathrm{v}_{3}$ is 1 as corresponding to the membership function of background noise. The rest can be gained in the same reason. The matrix $\mathrm{R}_{1}$ can be gained from those five membership functions and then compute $\mathrm{O}_{1}$ by formula (6). This detail of operation is shown as follows:

$$
\begin{aligned}
& \text { MS1's } \mathrm{O}_{1}=\mathrm{W}_{1} \times \mathrm{R}_{1} \\
& =\left[\begin{array}{lllll}
0.1 & 0.8 & 0.1 & 0 & 0
\end{array}\right]\left[\begin{array}{ccc}
0 & 0 & 1 \\
0.5 & 1 & 0 \\
0.2 & 0.6 & 0 \\
0 & 0 & 1 \\
0 & 0 & 1
\end{array}\right] \\
& =\left[\begin{array}{lll}
0.42 & 0.86 & 0.1
\end{array}\right]
\end{aligned}
$$

As above, the outcome shows that $\mathrm{o}_{11}$ is $0.42, \mathrm{o}_{12}$ is 0.86 , and $\mathrm{o}_{13}$ is 0.1 . Consequently, the logical expression of user's background are $\mathrm{O}_{12}>\mathrm{O}_{11} \wedge \mathrm{O}_{11}>\mathrm{O}_{13}$. According to the rule base in Table 2 , the estimate result is the "half-indoor". Accordingly, run, extremely busy, and extremely disorder are the estimate results of $\mathrm{O}_{2}, \mathrm{O}_{3}$ and $\mathrm{O}_{4}$, respectively. To sum up, the overall result $\mathrm{O}$ is as follows.

$$
\mathrm{O}=\{\mathrm{III}, \mathrm{IV}, \mathrm{VI}, \mathrm{VI}\}=\{\text { half-indoor, run, extremely busy, extremely disorder }\}
$$

The result $\mathrm{O}$ vector reveals that the user with MS1 is running at half-indoor environment. In addition, he/she is estimated to be busy and has disorder state now. For campus study, the student may be attending P. E. Class.

The example of a single mobile node is illustrated as above. We proceed to discuss the multinodes example. If a mobile user has a disorder tendency, the entertainment message may not be suitable for him/her at present. The mobile service provider will be able to know such situation by pay attention to the user situation $\left(\mathrm{O}_{4}\right)$. Supposed mobile node MS3 is taken for instance. Through the process of the membership functions and formula (6) discussed above, the results are generated as following.

$$
\text { MS3's } \mathrm{O}_{4}=\mathrm{W}_{4} \times \mathrm{R}_{3}
$$


International Journal of Database Management Systems (IJDMS) Vol.12, No.3, June 2020

$$
\begin{aligned}
& =\left[\begin{array}{lllll}
0.7 & 0 & 0.3 & 0.1 & 0.2
\end{array}\right]\left[\begin{array}{rrr}
0 & 0 & 1 \\
0 & 0 & 0.7 \\
0 & 0.2 & 0.7 \\
0 & 0 & 1 \\
1 & 0 & 0
\end{array}\right] \\
& =\left[\begin{array}{lll}
0.2 & 0.06 & 1
\end{array}\right]
\end{aligned}
$$

All results about five mobile nodes are as follows:

$$
\begin{aligned}
& \text { MS1's } \mathrm{O}_{4}=\left[\begin{array}{lll}
0.2 & 0.3 & 0.7
\end{array}\right], \\
& \mathrm{MS}^{2} \mathrm{~s} \mathrm{O}_{4}=\left[\begin{array}{lll}
0.3 & 0.5 & 0.33
\end{array}\right], \\
& \mathrm{MS}^{\prime} \mathrm{s} \mathrm{O}_{4}=\left[\begin{array}{lll}
0.2 & 0.06 & 1
\end{array}\right], \\
& \text { MS4's } \mathrm{O}_{4}=\left[\begin{array}{lll}
0.7 & 0 & 0
\end{array}\right], \\
& \mathrm{MS}^{\prime} \mathrm{s} \mathrm{O}_{4}=\left[\begin{array}{lll}
0.3 & 0.7 & 0.5
\end{array}\right] \text {. }
\end{aligned}
$$

Both of MS1 and MS3 have the lower o11 value and higher o13 value. They are considered to be in a similar situation. Additionally, the lower o11 value and higher o13 value imply that the users of MS1 and MS3 are the disorder tendency, so the entertainment advertising messages are not suitable for them and will be avoided to be sent in the present. It also can be estimated that the mobile user of MS4 may be at a fine situation without disturbance.

\subsection{Illustration for Weight Decision}

The weight decision method has been introduced in Section 4.3. If training data are available, we can use them to get the weight which is more reliable. For instance, the movement $\left(\mathrm{O}_{2}\right)$ is by vehicle and $\mathrm{O}_{2}$ is defined as $\left[\begin{array}{lll}0.3 & 0.2 & 0.8\end{array}\right]$ which conforms to the logical expression $\mathrm{O}_{13}>\mathrm{O}_{11} \wedge$ $\mathrm{O}_{11}>\mathrm{O}_{12}$. In addition, a matrix $\mathrm{R}_{3}$ for MS3 has been obtained as follows.

$$
R_{3}=\left[\begin{array}{ccc}
0 & 0 & 1 \\
0 & 0 & 0.7 \\
0 & 0.2 & 0.7 \\
0 & 0 & 1 \\
1 & 0 & 0
\end{array}\right]
$$

Suppose that there are three weight alternatives for $\mathrm{W}_{2}$, which are $\{0.2,0,0.8,0,0.5\},\{0,0.1$, $0.8,0.1,0.1\}$, and $\{0.3,0,0.4,0.2,0.6\}$. The three vectors are used to do fuzzy composition with R. Thus, the computing results are [ [ $\left.\begin{array}{llll}0.5 & 0.16 & 0.76\end{array}\right]$, [ $\left.\begin{array}{llll}0.1 & 0.16 & 0.73\end{array}\right]$, and [ [ $\left.\begin{array}{llll}0.6 & 0.08 & 0.78\end{array}\right]$ accordingly. Because $[0.10 .160 .73]$ does not conform to the logical expression $\mathrm{O}_{13}>\mathrm{O}_{11} \wedge \mathrm{O}_{11}>\mathrm{O}_{12}$, $\{0,0.1,0.8,0.1,0.1\}$ should not be considered. The approaching degree between $\left(\mathrm{O}_{\mathrm{i}}\right)_{\mathrm{t}}$ and $\mathrm{O}_{\mathrm{i}}$ can be computed via formula (7). These gained results are 0.85 and 0.97 respectively. The degree 0.97 is greater than 0.85 . Hence, the weight set candidate $\{0.3,0,0.4,0.2,0.6\}$ should be selected.

\subsection{Applied Scenarios}

Our research intends to discover more facts about users' surroundings and behavior. This proposed mechanism in the research is supposed to be applied to some coming mobile applications, such as mobile advertising. The mobile advertising has two major types including the pull marketing and the push marketing [13]. Both of them can adopt the information which is extended from our mechanism. Through the information derived from our mechanism, users' real-time needs can be probably explored and satisfied instantaneously. This research support the activity recognition required in the mobile context-aware services, which will help mobile 
service providers to give the proper services smartly. As you can imagine, it is useless to push the gas station message to a user walking outdoors. On the contrary, it may be suitable to show him the coupon of the ice cream store front.

In addition, the context-aware server will be able to collect real-world context data from multiple users in the scheme. Based on the analysis of these collected data, it becomes possible to develop a more reliable high-level context recognition mechanism. In other words, the service provider will be capable to distribute more accurate context-aware information and applications to mobile users. Moreover, the trustworthy weight sets can be decided by the training data and weight decision approach during implementation phase. Finally, the fuzzy logic approach owns features making it a pretty good performance for chaotic decision problems e.g. activity recognition with no need of precise, noise-free inputs. Hence, the proposed mechanism will make the contextaware applications more feasible and flexible.

\section{Conclusions}

The proposed scheme contributes a feasible way to discover the data of mobile device users and explore their surroundings. A fuzzy-based model is used to implement such application, because it can summarize the data from sensors to reason the users' contexts. The signal processing techniques and classification mechanism are also investigated. As a result, it will become possible to provide the real-time context-aware application to users by their situations accordingly.

One of the important challenges of providing mobile services is the accurate recognition of human activities. The technology enables service providers to determine a potential customer's activity and provide the appropriate context-aware services to him. A technical mechanism for the realization of the context awareness is provided in this paper. Furthermore, it can be utilized to recognize the basic information for inferring the characteristic identical or close to a user's situation. As long as other important mobile technologies progresses maturely, many amazing mobile services enhancing mobile commerce will become feasible and available. For instance, mobile users not only receive the instant promotion message as his are near to a department store, but also the interesting digital information at a tourist attraction, exhibition and etc.

To sum up, a context-aware mechanism serving an important part of providing mobile services is studied in this research. A reasoning scheme is discussed to illustrate how to estimate the users' situations analytically. Thus, more and more applications that satisfy users' immediate need could be developed in the near future, according to the concept revealed in this paper. Besides, based on the proposed mechanism, novel mobile services will have more diversity and offer more chance to enhance mobile commerce with feasibility and flexibility.

\section{REFERENCES}

[1] Omer B. Sezer, E. Dogdu, and Ahmet M. Ozbayoglu (2018) Context-Aware Computing, Learning, and Big Data in Internet of Things: A Survey, IEEE Internet of Things, vol. 5, no. 1, pp. 1-27

[2] Petcovici A. and Stroulia E. (2016) Location-based services on a smart campus: A system and a study, Proceedings of IEEE the 3rd World Forum on Internet of Things, pp. 94-99

[3] Marek R. Ogiela and Lidia Ogiela (2013) Cognitive Information Systems in Ubiquitous Computing, Proceedings of the 7th International Conference on Innovative Mobile and Internet Services in Ubiquitous Computing, vol. 43, no. 2, pp. 246-248

[4] P. Korpipaa, J. Mantyjarvi, J. Kela, H. Keranen, and E.J. Malm (2003) Managing Context Information in Mobile Devices, IEEE Pervasive Computing, vol. 2, no. 3, pp. 42-51

[5] M. Mühlenbrock, O. Brdiczka, D. Snowdon, and J.-L. Meunier, (2004) Learning to Detect User 
International Journal of Database Management Systems (IJDMS) Vol.12, No.3, June 2020

Activity and Availability from a Variety of Sensor Data, Proceedings of the Second IEEE Annual Conference on Pervasive Computing and Communications, pp. 13-22

[6] Gorka Azkune and Aitor Almeida (2018) A Scalable Hybrid Activity Recognition Approach for Intelligent Environments, IEEE Access, vol. 6, pp. 41745-41759

[7] F. Nocera, M. Mongiello, A. Parchitelli, E. Di Sciascio, and L. Patrono (2019) A model for Reflective Middleware based on fuzzy rule for context-awareness injection in ubiquitous computing environments, Proceedings of 4th International Conference on Smart and Sustainable Technologies, pp. 1-7

[8] Liqin Guo, Lixue Wang, Yongyi He, and Sheng Bao (2017) Navigation System of the Stacking Vehicle Based on Fuzzy Control and Laser Scanner, Proceedings of 10th International Symposium on Computational Intelligence and Design, vol. 2, pp. 97-102

[9] Sadik K. Gharghan, R. Nordin, Aqeel M. Jawad, Haider M. Jawad, and M. Ismail (2018) Adaptive Neural Fuzzy Inference System for Accurate Localization of Wireless Sensor Network in Outdoor and Indoor Cycling Applications, IEEE Access, vol. 6, pp. 38475-38489

[10] Dianshuang Wu, Guangquan Zhang, and Jie Lu, (2015) A Fuzzy Preference Tree-Based Recommender System for Personalized Business-to-Business E-Services, IEEE Transactions on Fuzzy Systems, vol. 23, no. 1, pp. 29-43

[11] Al-Qerem Ahmad, (2014) "Performance Evaluation of Transaction Processing in Mobile Data Base Systems", International Journal of Database Management Systems, vol. 6, no. 2, pp. 19-38.

[12] Harikesh B. Yadav and Dilip K. Yadav, (2015) "A method for generating membership function from numerical data", Journal of Intelligent \& Fuzzy Systems, vol. 29, pp. 2227, 2015.

[13] Ruibing Wang, Qinglong Gou, Tsan-Ming Choi, and Liang Liang (2018) Advertising Strategies for Mobile Platforms With "Apps", IEEE Transactions on Systems, Man, and Cybernetics: Systems, vol. 48 , no. 3 , pp. $757-778$

\section{AUTHORS}

Kuen-Liang Sue received the M.S. degree in computer science and information engineering and the Ph.D. degree in information management from National Chiao Tung University, Hsinchu, Taiwan. He is currently an assistant professor with the Department of Information Management, National Central University, Zhongli, Taiwan. His research topics include mobile computing, IoT, and network security, etc.

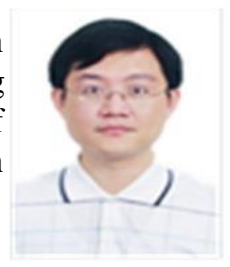

